Rapid Reviews COVID-19

\title{
Review 2: "Seroprevalence of SARS-COV-2 Antibodies in Scottish Healthcare Workers"
}

\section{Tatjana Vilibić-Čavlek ${ }^{1}$, Vladimir Savic ${ }^{2}$}

${ }^{1}$ Croatian Institute of Public Health: Hrvatski zavod za javno zdravstvo, Department of Virology, Croatia,

${ }^{2}$ Croatian Veterinary Institute: Hrvatski veterinarski institut

Published on: Dec 17, 2020

DOI: $10.1162 / 2 \mathrm{e} 3983 f 5.1 \mathrm{a} 331 \mathrm{e} 81$

License: Creative Commons Attribution 4.0 International License (CC-BY 4.0). 


\section{$\underline{\text { RR:C19 Evidence Scale rating by reviewer: }}$}

- Reliable. The main study claims are generally justified by its methods and data. The results and conclusions are likely to be similar to the hypothetical ideal study. There are some minor caveats or limitations, but they would/do not change the major claims of the study. The study provides sufficient strength of evidence on its own that its main claims should be considered actionable, with some room for future revision.

\section{Review:}

We have read with interest the manuscript entitled "Seroprevalence of SARS-CoV-2 Antibodies in Scottish Healthcare Workers" by Abo-Leyah, et al. In this prospective observational study, the authors analyzed the seroprevalence of SARS-CoV-2 infection in 2062 health and social care workers employed in the National Health Service in Tayside (Scotland, United Kingdom). A random selection of blood samples taken at General Practice Surgeries were tested as the MATCH study cohort. For detection of SARS-CoV-2 antibodies, a commercial chemiluminescent immunoassay (CLIA) method that detects antibodies against the receptor-binding domain (RBD) of the SARS-CoV-2 spike (S1) protein was used.

The results of the study showed that healthcare workers (HCW) were three times more likely to test SARS-CoV-2 positive than general population. The overall seroprevalence was $14.5 \%$ and varied significantly among HCW subgroups from $8.6 \%$ to $26 \%$ compared to $4.5 \%$ in the Scottish general population. Dentistry staff was identified as a subpopulation at the highest risk for COVID-19 (seroprevalence 26\%), followed by health care assistants (seroprevalence 23.3\%). The presented results also demonstrated that a significant proportion of SARS-CoV-2 seropositive HCW (18.5\%) were asymptomatic.

There are some minor limitations of the study, but they do not change the major claims of the study. Although several limitations are discussed, one additional limitation needs to be addressed. Since only CLIA test was used for the antibody detection without confirmatory neutralization tests (neither surrogate ELISA neutralization test nor virus neutralization test in a cell culture), potential crossreactivity with other coronaviruses should not be excluded with certainty.

Since the HCW are a population with an increased risk for SARS-CoV-2 infection, the seroprevalence data provides information on the extent of the disease (especially among asymptomatic cases who may potentially transmit the virus to their colleagues and patients) and contributes to a better understanding of the epidemiology of COVID-19 in this population group. In addition, the 
seroprevalence data identified in this large-scale seroepidemiological study will be useful to raise awareness and, thereby, protect HCW during the future waves of COVID-19 pandemic. 